\title{
Gestão de Custos com Comunicação Integrada de Marketing nas Clínicas de Fisioterapia e Estética no Município de Piripiri-PI
}

\author{
Laise Do Nascimento Silva \\ Graduação em Administração em andamento pelo Instituto Federal de Educação, \\ Ciência e Tecnologia do Piauí - IFPI \\ Rua Euclides Cordeiro, N 90. Centro, Piripiri. Piauí. CEP: 64260-000 \\ E-mail: laisenascimento1996@gmail.com
}

Elane Dos Santos Silva Barroso Mestrado em Administração e Controladoria pela Universidade Federal do Ceará - UFC Professora do Instituto Federal de Educação, Ciência e Tecnologia do Piauí - IFPI Av. Zequinha Freire, 375. Bairro Santa Isabel. Teresina/Piauí. CEP: 64053-400

E-mail: elane.silva@ifpi.edu.br

Geane Maria Oliveira Lima Graduação em Administração em andamento pelo Instituto Federal de Educação, Ciência e Tecnologia do Piauí - IFPI Rua Francisco Castro, 80. Coehb. Batalha/PI. CEP: 64190-000 E-mail: geanerosariana@gmail.com

Jane Gabriela Soares de Lemos Graduação em Medicina Veterinária pela Universidade Federal do Piauí - UFPI Professora do Instituto Federal de Educação, Ciência e Tecnologia do Piauí - IFPI Rua Professora Raimunda Mendes, 1040. Centro. Piripiri. Piauí. CEP: 64260-000 E-mail: lemos.jane@yahoo.com.br

Edvaldo Pio da Silva Graduação em Administração em andamento pelo Instituto Federal de Educação, Ciência e Tecnologia do Piauí - IFPI Gestor na Empresa Lili Doces BR 343, KM 190. Zona Rural, S/N. Piripiri. Piauí. CEP: 64260-000 E-mail: edvaldop.dasilva@hotmail.com

\section{RESUMO}

A Comunicação Integrada de Marketing (CIM) tem sido bastante valorizada e utilizada nos planos estratégicos das empresas visto que potencializa o alcance dos objetivos, fornece maior conexão com o cliente e eleva os resultados de venda. Mas, para que ocorra o equilíbrio organizacional também é essencial a contabilidade de custos. Nesse sentido, a presente pesquisa levantou como problemática: Os gestores das clínicas de fisioterapia e estética do munícipio de Piripiri identificam a Comunicação Integrada de Marketing como despesa ou investimento? Objetivou-se de forma geral averiguar a 
Gestão de Custos com Comunicação Integrada de Marketing nas Clínicas de Fisioterapia e Estética no Município de Piripiri-PI Laise Do Nascimento Silva, Elane Dos Santos Silva Barroso, Geane Maria Oliveira Lima, Jane Gabriela Soares de Lemos, Edvaldo Pio da Silva

concepção que os gestores dessas clínicas têm sobre a CIM, levando em consideração sua classificação como despesa ou investimento. E especificamente, identificar o perfil das clínicas de fisioterapia e estética em Piripiri-PI; averiguar se existem o uso de ferramentas para controle de custos e quais elementos da CIM são empregados por essas empresas. Foi aplicado um questionário semiestruturado contendo 27 questões para os gestores das empresas. A pesquisa apontou que $60 \%$ dos empresários fazem seu próprio controle de custo e apenas $20 \%$ têm o auxílio de um contador. Em relação ao marketing integrado os dados mostraram que $80 \%$ conhecem razoavelmente e $20 \%$ não apresentam nenhum conhecimento, mas, visualizam os gastos com essa ferramenta como um investimento. $60 \%$ utiliza a propaganda, $20 \%$ a publicidade e 0 marketing direto. Conclui-se que existe a necessidade de conhecimento para a tomada de decisões em relação ao controle de custos.

Palavras-chave: Comunicação Integrada de Marketing. Despesa. Investimento. Clínicas.

\section{Cost Management With Integrated Marketing Communication in Physiotherapy and Aesthetics Clinics in the City of Piripiri-PI}

\section{ABSTRACT}

Integrated Marketing Communication (CIM) has been highly valued and used in companies' strategic plans. It enhances objectives, provides a greater connection with the customer, and increases sales results. But, for organizational balance to occur, cost accounting is also essential. In this sense, the present research has raises the following question: Do the physiotherapy and aesthetics clinics' managers identify Integrated Marketing Communication as an expense or investment in the municipality of Piripiri? The general objective is to find outthe conception these clinics' managers have about CIM if it is an expense or an investment. Also, it was intended to identify the profile of physiotherapy and aesthetic clinics in Piripiri-PI, that is, would they be working with any tools for cost control and which of these companies have already used CIM elements. A semi-structured questionnaire, containing 27 questions, was applied for company managers. The survey showed that $60 \%$ of businesspeople do their own cost control, and only $20 \%$ have a professional accountant's support. The data showed that $80 \%$ know reasonably about integrated marketing, and $20 \%$ do not know, but they consider spending money on this tool as an investment. $60 \%$ use advertising, and $20 \%$ use both: advertising and direct marketing. It is concluded that there is a need for knowledge to make decisions regarding cost control.

Key-words: Integrated Marketing Communication. Expense. Investment. Clinics. 
Gestão de Custos com Comunicação Integrada de Marketing nas Clínicas de Fisioterapia e Estética no Município de Piripiri-PI Laise Do Nascimento Silva, Elane Dos Santos Silva Barroso, Geane Maria Oliveira Lima, Jane Gabriela Soares de Lemos, Edvaldo Pio da Silva

\section{Gestión de Costos con Comunicación de Marketing Integrada en Clínicas de Fisioterapia y Estética em la Ciudad de Piripiri-PI}

\section{RESUMEN}

La Comunicación Integrada de Marketing (CIM) ha sido muy valorada y utilizada en los planes estratégicos de las empresas ya que potencia el logro de objetivos, proporciona una mayor conexión con el cliente y aumenta los resultados de ventas. Pero, para que ocurra el equilibrio organizacional, la contabilidad de costos también es esencial. En este sentido, esta investigación planteó como problemática: ¿Los gerentes de las clínicas de fisioterapia y estética en el municipio de Piripiri identifican la Comunicación Integrada de Marketing como un gasto o inversión? El objetivo general fue conocer la concepción que los gerentes de estas clínicas tienen sobre el CIM, teniendo en cuenta su clasificación como gasto o inversión. Y en concreto, identificar el perfil de las clínicas de fisioterapia y estética en Piripiri-PI; averigüe si existen herramientas para el control de costos y qué elementos de CIM son empleados por estas empresas. Se aplicó un cuestionario semiestructurado que contenía 27 preguntas para directivos de empresas. La encuesta mostró que el $60 \%$ de los empresarios hacen su propio control de costos y solo el $20 \%$ cuenta con la ayuda de un contador. En relación al marketing integrado, los datos mostraron que el $80 \%$ sabe razonablemente y el $20 \%$ no tiene ningún conocimiento, pero ven el gasto en esta herramienta como una inversión. El $60 \%$ utiliza publicidad, el $20 \%$ utiliza publicidad y marketing directo. Se concluye que existe la necesidad de conocimiento para tomar decisiones sobre el control de costos.

Palabras clave: Comunicación de marketing integrada. Gastos. Inversión. Clínicas.

\section{INTRODUÇÃO}

O mercado consumidor tem sido cada vez mais exigente, fato esse que contribui para o aumento da competitividade entre as organizações. E, para se manter em um ambiente concorrido as empresas buscam comunicar-se com seu público-alvo a fim de continuarem presentes na mente dos consumidores e conseguir atrair novos clientes.

A Comunicação Integrada de Marketing (CIM) tem sido bastante valorizada e utilizada nos planos estratégicos das organizações. Isso porque apresenta-se como uma ferramenta capaz de unificar e ampliar as atividades das empresas ao tempo em que oportuniza um relacionamento mais sólido e frequente dessas com seus clientes (Kotler \& Keller, 2010). 
Gestão de Custos com Comunicação Integrada de Marketing nas Clínicas de Fisioterapia e Estética no Município de Piripiri-PI Laise Do Nascimento Silva, Elane Dos Santos Silva Barroso, Geane Maria Oliveira Lima, Jane Gabriela Soares de Lemos, Edvaldo Pio da Silva

Nesse sentido, entende-se como comunicação integrada a unificação de todas as formas de comunicação e suas respectivas mensagens transmitidas pela empresa com o mesmo objetivo (Silva, Pitombo, \& Zaccaria, 2017). Entre os elementos que a compõem encontram-se: propaganda, marketing direto, publicidade, promoção de eventos, merchandising, internet, ponto-de-venda, vendas, entre outros. Vale considerar que a Comunicação Integrada de Marketing e o desenvolvimento organizacional dependem da eficácia de outras áreas do conhecimento, entre elas a contabilidade, que nesta pesquisa está associada aos custos (Sarquis, Hoeckesfeldet, Cittadin, \& Lenzi, 2019).

A contabilidade de custos é uma ferramenta útil no gerenciamento, controle de recursos, assim como fonte de informações necessárias para a tomada de decisão. Ainda, integra os gastos que de maneira abrangente se dividem em custos, gastos relacionados à produção, despesas, gastos voltados para atividades administrativas e investimentos, gastos aplicados que geram retorno para a organização.

De acordo com dados nacionais do Sebrae (2016), o número de registros de microempreendedores individuais (MEls) no segmento de fisioterapia e estética teve um incremento de 567\% passando de 72.309 para 482.455 em janeiro de 2016 . Outro fator que justifica esse crescimento está na participação feminina no mercado de trabalho e no seu poder de compra. Segundo o levantamento do Cadastro Geral de Emprego e Desemprego (CAGED) divulgados em 2017, as mulheres compõem $44 \%$ das vagas no mercado de trabalho formal. Além disso, são as que mais intervém no aumento de vendas nesse segmento (Exame, 2017).

As clínicas de fisioterapia e estética são segmentos que vêm crescendo devido ao aumento no número de profissionais atuantes nesse mercado e a busca de bemestar por parte da população. Considerando a ascensão nesse setor e sua presença no município de Piripiri, no Piauí, levantou-se como problemática: Os gestores das clínicas de fisioterapia e estética do munícipio de Piripiri identificam a Comunicação Integrada de Marketing como despesa ou investimento? 
Gestão de Custos com Comunicação Integrada de Marketing nas Clínicas de Fisioterapia e Estética no Município de Piripiri-PI Laise Do Nascimento Silva, Elane Dos Santos Silva Barroso, Geane Maria Oliveira Lima, Jane Gabriela Soares de Lemos, Edvaldo Pio da Silva

Assim sendo, objetivou-se com a referida pesquisa de maneira geral, averiguar a concepção que os gestores dessas clínicas têm sobre a Comunicação Integrada de Marketing, levando em consideração sua classificação como despesa ou investimento. E, especificamente, identificar o perfil das clínicas de fisioterapia e estética em Piripiri$\mathrm{PI}$; averiguar se existem o uso de ferramentas para controle de custos e quais elementos da Comunicação Integrada de Marketing são utilizados por essas empresas.

Dessa forma, a expressividade deste trabalho é garantida através das contribuições que esse estudo trará para o meio acadêmico e social ao introduzir reflexões acerca da importância da Comunicação Integrada de Marketing para o sucesso empresarial, assim como evidenciar as práticas desenvolvidas pelos empresários situados no município de Piripiri que operam no mercado de bem-estar e estética. Tais evidências ajudarão estudantes, docentes e a sociedade a compreenderem os desafios da administração enfrentados por gestores sem uma formação acadêmica.

\section{COMUNICAÇÃO INTEGRADA DE MARKETING E A GESTÃO DE CUSTOS}

A Comunicação Integrada de Marketing participa do composto de Marketing e representa uma ferramenta essencial no âmbito das organizações visto que através desse instrumento as empresas conseguem dialogar com o mercado e alcançar posições satisfatórias, denotando a importância da CIM para quem a utiliza.

\subsection{Comunicação Integrada de Marketing}

O marketing relaciona-se a uma área administrativa especializada em estudar, criar e gerenciar relacionamentos com o mercado-alvo das organizações visando construir uma marca representativa e competitiva. Ou seja, objetiva conhecer o comportamento do mercado dos clientes e das organizações.

Até os anos de 1950 a única atribuição do marketing era fazer com que a produção excedente das fábricas fosse interessante ao seu público-alvo (Merabet, 
Gestão de Custos com Comunicação Integrada de Marketing nas Clínicas de Fisioterapia e Estética no Município de Piripiri-PI Laise Do Nascimento Silva, Elane Dos Santos Silva Barroso, Geane Maria Oliveira Lima, Jane Gabriela Soares de Lemos, Edvaldo Pio da Silva

Silva, Gonçalves, \& Barros, 2020). Ademais, com as transformações culturais, sociais e os avanços tecnológicos o marketing ganhou uma nova roupagem, uma abordagem diferenciada que colocava como prioridade o cliente e não mais o produto no qual o foco estava em gerar valor para o cliente, isto é, fazer com que ele perceba que está a adquirir um produto ou serviço que vale muito mais do que o preço estabelecido (Barboza \& Silva, 2019).

Entre as principais atribuições do marketing estão o ato de criar um produto de acordo com as necessidades do cliente, precificá-lo estrategicamente; gerir a distribuição e promover esses mesmos produtos e serviços (Diniz \& Suarez, 2018). Acrescenta-se ainda que as decisões convergentes a essas atribuições devem ser ponderadas levando em consideração as variáveis dos ambientes internos e externos do mercado. O externo, no geral, abrange seis componentes: ambiente econômico, ambiente demográfico, ambiente natural, ambiente tecnológico, ambiente político-legal e ambiente sociocultural (Pereira, 2015). O ambiente interno, por sua vez, é composto pelos colaboradores, distribuidores, fornecedores e clientes (Pereira, 2015).

Partindo disso, ressalta-se que existem variadas formas e canais por meio dos quais pode se promover a venda de um produto ou serviço, por exemplo, através da publicidade, venda pessoal, relações públicas, promoção e merchandising (Merabet et al., 2020). Entende-se como comunicação integrada à junção de estratégias que objetivam atingir um público-alvo a partir dos esforços de marketing, sendo a CIM uma ferramenta participante do estudo do mercado. Em outras palavras, representa a unificação de todas as formas de comunicação e suas respectivas mensagens transmitidas pela empresa com o mesmo objetivo (Breda, Cruz, Hermes, \& Medeiros, 2020).

O termo Comunicação Integrada de Marketing designa 0 ato de uniformizar a mensagem persuasivamente propagada pela marca, de modo que todos os canais que a organização utiliza para promover sua marca, seus produtos e serviços sinalizem o mesmo posicionamento na mente dos clientes (Petrin, Cardozo, \& Christino, 2019). 
Gestão de Custos com Comunicação Integrada de Marketing nas Clínicas de Fisioterapia e Estética no Município de Piripiri-PI Laise Do Nascimento Silva, Elane Dos Santos Silva Barroso, Geane Maria Oliveira Lima, Jane Gabriela Soares de Lemos, Edvaldo Pio da Silva

Cada canal possui um público diferenciado e requer uma estratégia diferente. As relações públicas e de publicidade, por exemplo, mais executadas em jornais de rádio, televisão e eventos direcionam a marca para o público de massa, enquanto a venda pessoal se conecta diretamente com o público que, de algum modo, já demonstrou interesse em consumir os produtos e serviços os quais a organização oferece (Petrin et al., 2019).

Entende-se como propaganda a forma paga e impessoal da comunicação de marketing que busca atingir o público-alvo através das ferramentas da comunicação e com base em seu posicionamento perante o consumidor. Esse item pode ser entendido como um elemento que de maneira pecuniária auxilia na apresentação do produto ou serviço de uma empresa, utilizando-se de um anunciante para sua exposição junto à sociedade (Seta, Mainardes, \& Silva, 2016). A publicidade na perspectiva de Seta et al. (2016) corresponde às práticas ativas direcionadas ao mercado que oportuniza alcançar visibilidade das empresas e seus produtos ou serviços para os consumidores potenciais.

Com relação ao item merchandising Kotler e Keller (2010) o definem como informação do produto ou serviço por meio do rádio, televisão ou internet. A comunicação pela internet compreende nesse aspecto um conjunto de atividades interativas com os clientes por meio de sites, e-mails, redes sociais, entre outros.

Além desses canais infere-se que a internet representa um meio de comunicação com o qual as empresas devem contar, principalmente diante de crises econômicas que as motivam a tomarem medidas que necessitarão do uso de canais digitais. As organizações terão de investir em estruturas de comunicação e nos ambientes digitais, posto que lançar produtos sem trazê-los ao conhecimento de possíveis compradores minimizarão a permanência da empresa no mercado em tempos de crise (Silva et al., 2017).

A esse respeito, enfatiza-se que o consumidor da era da informação está mais criterioso no seu processo de decisão de compra. Esse costuma comprar somente das marcas que conhece. Corroborando com esse pensamento, Seta et al. (2016) afirmam 
Gestão de Custos com Comunicação Integrada de Marketing nas Clínicas de Fisioterapia e Estética no Município de Piripiri-PI Laise Do Nascimento Silva, Elane Dos Santos Silva Barroso, Geane Maria Oliveira Lima, Jane Gabriela Soares de Lemos, Edvaldo Pio da Silva

que as marcas devem gerar um posicionamento na mente dos clientes de forma que não somente voltem a consumir produtos de determinado segmento, mas, construir um canal que possa propagar as qualidades e o valor da marca.

No entanto, algumas organizações não usufruem plenamente dos canais mais acessados pelo seu público-alvo. Ou seja, o esforço empregado em veículos de comunicação em massa pode não ser tão eficaz e eficiente se a organização não direciona a comunicação para canais segmentados de acordo com o perfil do público que pretende atingir (Breda et al., 2020).

Assim, torna-se possível, por exemplo, que uma propaganda sobre seções de fisioterapia para atletas consiga atingir as pessoas realmente interessadas, sendo veiculado mensagens nas redes sociais. A distribuição de anúncios na internet segmenta o perfil do público que o anunciante pretende alcançar, sendo que o uso de mídias sociais auxilia na propagação de anúncios para alcançar pessoas que possuem interesse em determinada marca (Silva et al., 2020). Logo, a utilização de um canal de comunicação requer investimentos financeiros, uma vez que estabelece contato direto com o público e dispõe de meios para inserir links de produtos ou serviços, por exemplo.

Em suma, a comunicação Integrada tem se mostrado cada dia mais relevante para os resultados de uma organização, sendo capaz de posicionar fortemente a marca no imaginário das pessoas, reforçando em todos os canais possíveis o seu valor e utilidade (Garcia \& Mainardes, 2016).

Vale destacar que muitas organizações podem enfrentar desafios para a efetiva implantação de um canal de comunicação, já que adquiri-lo requer uma articulação de todos os departamentos da empresa (Queiroz, Queiroz, Yanaze, \& Maziere, 2018). O planejamento das ações de marketing deve ser feito de forma coerente com apoio e envolvimento de todos os setores das organizações, avaliando planos estratégicos, lucros pretendidos, balanços e demonstrativos de resultados de ações efetuadas.

Nesse contexto, compreende-se que a aquisição de ferramentas para comunicação requer a disposição de recursos para sua consolidação. Daí, a conexão 
Gestão de Custos com Comunicação Integrada de Marketing nas Clínicas de Fisioterapia e Estética no Município de Piripiri-PI Laise Do Nascimento Silva, Elane Dos Santos Silva Barroso, Geane Maria Oliveira Lima, Jane Gabriela Soares de Lemos, Edvaldo Pio da Silva

entre o marketing e a contabilidade de custos, visto que se responsabiliza pelo gerenciamento financeiro de toda organização. No que diz respeito à contabilidade de custos, a mesma tem como objetivo fornecer informações para todos os níveis gerenciais de uma entidade, auxiliando no planejamento e controle das operações e de tomada de decisões (Queiroz et al., 2018).

\subsection{A Importância da Gestão de Custos para as Organizações}

A necessidade de conhecer e controlar os custos dos produtos e serviços são imprescindíveis para toda e qualquer empresa, independentemente de seu porte estrutural e financeiro. Primeiro passo consiste em entender que custos são gastos relacionados a produtos e serviços participantes da confecção de outros bens e serviços finais (Lopes \& Beuren, 2017). Em outras palavras, todo valor investido na produção e que está diretamente ligado a atividade principal de uma empresa deve ser considerado como um custo.

As informações constantes nas operações financeiras das organizações devem ser claras, precisas e de acordo com a realidade para que a contabilidade financeira possa planejar melhor o seu desembolso. Já a contabilidade gerencial controla o que está sendo produzido, procurando reduzir o máximo de custos e auxiliar para tomada de decisões mais assertivas (Antônio, Rathke, Moraes, \& Ambrozini, 2018). Ou seja, a contabilidade deve fornecer aos administradores informações úteis, oportunas e fidedignas para que o administrador possa fazer uso na tomada de decisão.

Os acontecimentos operacionais diários necessitam ser registrados e estarem disponíveis para os membros da organização, uma vez que os registros dos processos auxiliam no controle dos recursos que entram e saem da empresa, sendo hábeis na tomada de decisão agregados aos objetivos principais da organização (Marcolin \& Deus, 2018).

Bergmann, Gresele e Walter (2019) descreve que custo corresponde ao esforço financeiro despendido para a aquisição de um bem ou serviço necessário para a 
Gestão de Custos com Comunicação Integrada de Marketing nas Clínicas de Fisioterapia e Estética no Município de Piripiri-PI Laise Do Nascimento Silva, Elane Dos Santos Silva Barroso, Geane Maria Oliveira Lima, Jane Gabriela Soares de Lemos, Edvaldo Pio da Silva

operacionalização das atividades da organização de forma que os seus objetivos possam ser alcançados no que tange a concepção de produtos.

Crepaldi e Crepaldi (2018) afirmam que o custo se diferencia da despesa, visto que representa o valor dos bens ou serviços não relacionados diretamente com a produção de outros bens ou serviços consumidos em um determinado período. A despesa gera um desembolso de ativos ou uma obrigação com terceiros e é registrada diretamente como uma conta redutora do Patrimônio Líquido. O custo se associa a tudo aquilo atrelado a obtenção de bens e serviços englobando os custos e despesas enquanto as despesas correspondem a dispêndios de cunho mais administrativo. Geralmente, tais definições são comuns no âmbito empresarial devido seu apoio contábil já que constam no balanço financeiro com foco na tomada de decisão.

$\mathrm{Na}$ visão de Crepaldi e Crepaldi (2018), as despesas relacionam-se a um custo participante da receita de um período contábil efetuado com algo equivalente a atividades secundárias e de cunho administrativo, mas que possuem probabilidade de gerar novas receitas.

Através desses conceitos, percebe-se que um mesmo bem ao longo da cadeia produtiva pode ser qualificado em determinada categoria passando a outra no estágio seguinte de produção, ou mesmo estar incluído em mais de uma categoria. E, como esses bens, os gastos relacionados à Comunicação Integrada de Marketing participam dessa cadeia, ora como despesa quando há a entrega do numerário e investimento quando há retorno para a organização.

\section{METODOLOGIA}

Para fins de execução dos objetivos elencados o referido estudo fez uso da pesquisa bibliográfica como base para o referencial teórico. Classifica-se como descritiva visto que visa descrever as características de determinada população ou fenômenos. Uma de suas qualidades está na utilização de técnicas padronizadas de coleta de dados, semelhantes a questionário e a observação sistemática (Gil, 2017). 
Gestão de Custos com Comunicação Integrada de Marketing nas Clínicas de Fisioterapia e Estética no Município de Piripiri-PI Laise Do Nascimento Silva, Elane Dos Santos Silva Barroso, Geane Maria Oliveira Lima, Jane Gabriela Soares de Lemos, Edvaldo Pio da Silva

Quanto ao procedimento técnico classifica-se como pesquisa de campo no qual na visão de Figueiredo e Souza (2011) "consiste na observação espontânea dos fatos ou fenômenos, geralmente no próprio local onde ocorrem tais fenômenos". Para tanto, o instrumento empregado no estudo correspondeu a um questionário semiestruturado contendo 27 questões objetivas sendo aplicado aos gestores das empresas entrevistadas. O questionário contou com perguntas que envolveram o perfil das empresas, o emprego da Comunicação Integrada de Marketing e Contabilidade de Custos.

Este estudo teve como objeto as clínicas de fisioterapia e estética na cidade de Piripiri, no Piauí localizado à $150 \mathrm{~km}$ da capital Teresina, em um universo de 06 empresas de pequeno porte, com uma amostra de 05 empresas. Ambas estão situadas no centro da cidade e atuam no setor de serviços. $\mathrm{Na}$ aplicação do questionário foi utilizado a ferramenta google forms. E para a tabulação e geração dos gráficos finais, fez-se uso do programa Excel 2016.

\section{ANÁLISE E DISCUSSÃO DOS RESULTADOS}

Este tópico traz a discussão dos principais resultados alcançados no estudo. Os dados iniciais constam informações sobre o perfil das clínicas e seus respectivos gestores participantes quanto ao sexo, tempo de trabalho, público-alvo e a margem de lucro.

Assim, no que se refere ao tempo de trabalho, verifica-se que $60 \%$ possuem entre 05 a 10 anos, 20\% entre 01 a 03 anos e 03 a 05 anos. Sendo que $80 \%$ dos participantes são mulheres e apenas $20 \%$ são homens e $40 \%$ tem como público-alvo as mulheres e pessoas que precisem de reabilitação e $20 \%$ declararam ser o público em geral.

Analisa-se que mesmo sendo do mesmo ramo de atividade as clínicas apresentam público-alvo distintos. O público-alvo em sua maioria são mulheres, isso se dá devido ao crescimento na participação feminina, no mercado de trabalho e do seu 
Gestão de Custos com Comunicação Integrada de Marketing nas Clínicas de Fisioterapia e Estética no Município de Piripiri-PI Laise Do Nascimento Silva, Elane Dos Santos Silva Barroso, Geane Maria Oliveira Lima, Jane Gabriela Soares de Lemos, Edvaldo Pio da Silva

potencial poder de compra. Com relação ao lucro, afirma-se que $60 \%$ possuem 10 a $15 \%$ de lucro, enquanto os $40 \%$ restantes declararam que o lucro chega a ser acima de $15 \%$. A lucratividade distinta pode ser reflexo do tempo de atuação dessas empresas no mercado.

A figura 01 a seguir notifica acerca do nível de conhecimento dos gestores das clínicas quanto à Comunicação Integrada de Marketing, os itens mais utilizados e as possíveis perdas pelo não uso da CIM:

\begin{tabular}{|c|c|c|c|c|c|}
\hline Conhecimento da CIM & $\%$ & $\begin{array}{c}\text { Itens da CIM mais } \\
\text { utilizados }\end{array}$ & $\%$ & $\begin{array}{c}\text { Perdas pelo não uso da } \\
\text { CIM }\end{array}$ & $\%$ \\
\hline Pouco & $0 \%$ & Propaganda & $60 \%$ & Clientes & $40 \%$ \\
\hline Razoável & $80 \%$ & Marketing Direto & $20 \%$ & Redução das Vendas & $40 \%$ \\
\hline Nenhum & $20 \%$ & Publicidade & $20 \%$ & Mercado e Clientes & $20 \%$ \\
\hline
\end{tabular}

Figura 1. Dados da Comunicação Integrada de Marketing

Fonte: Dados da Pesquisa (2020).

De acordo com a figura $01,80 \%$ dos gestores conhecem razoavelmente a definição da Comunicação Integrada de Marketing (CIM), ou seja, possuem um entendimento prévio da temática apresentada, divergindo dos $20 \%$ que afirmam não ter nenhum conhecimento. Declara-se que a ausência de conhecimento sobre esses elementos pode implicar em uma baixa eficácia nas atividades da organização e trazer possíveis perdas.

Define-se CIM como uma ferramenta que contempla todas as maneiras das empresas de entrarem em contato com o seu público. Propõe suprir as necessidades das empresas e garantir que as estratégias mercadológicas ocorram de forma eficaz ao possibilitar a interação entre essas junto ao mercado por meio da aplicação de itens como promoção, publicidade, propaganda entre outras que são partes da comunicação integrada e que refletem no resultado operacional. 
Gestão de Custos com Comunicação Integrada de Marketing nas Clínicas de Fisioterapia e Estética no Município de Piripiri-PI Laise Do Nascimento Silva, Elane Dos Santos Silva Barroso, Geane Maria Oliveira Lima, Jane Gabriela Soares de Lemos, Edvaldo Pio da Silva

A Comunicação Integrada de Marketing é de suma importância para que a empresa se aproxime do mercado-alvo, de forma que a transmissão de suas mensagens ocorra eficazmente. $O$ uso dessa ferramenta oportuniza vantagens competitivas além da obtenção de posicionamento e redução de gastos desnecessários (Petrin et al., 2019).

No que se refere aos itens da CIM, 60\% utiliza a propaganda e $20 \%$ a publicidade e o marketing direto. Constata-se que as empresas respondentes utilizam apenas alguns elementos isolados da CIM com o intuito de promover sua imagem de forma a alcançar lucratividade, participação e implementações estratégicas. Tanto a publicidade quanto a propaganda e o marketing direto propõem anunciar mensagens para promover a marca da empresa.

A Comunicação Integrada de Marketing corresponde a um conjunto de possibilidades que as empresas se utilizam para se comunicarem com seu público-alvo ou atrair mais clientes. Como comentado anteriormente, as mais destacadas pelas clínicas foram a propaganda, publicidade e o marketing direto. Empresas que não têm o conhecimento adequado sobre a CIM podem comprometer seus resultados, todavia, as que apresentam o conhecimento sobre a CIM mas não o praticam, então perdem a oportunidade de se destacarem no mercado.

Os participantes do estudo realçaram que a falta de uma comunicação eficiente traz perdas no que tange aos clientes, há uma redução das vendas e consequentemente perda de mercado. Os dados apresentados inferem que $40 \%$ dos respondentes consideraram perder os clientes e a redução nas vendas como prejuízos que podem ocorrer pela falta dessa estratégia de comunicação.

Essa situação gera impactos negativos nos resultados das empresas, pois perder cliente e não conseguir fidelizá-lo traz sérios danos para as entidades, demonstrando uma falta de administração. Sendo que $20 \%$ dos respondentes disseram que as perdas seriam no mercado e clientes, ou seja, a falta de informação a respeito do serviço que oferecem promoções e novidades interferem nas vendas. 
Gestão de Custos com Comunicação Integrada de Marketing nas Clínicas de Fisioterapia e Estética no Município de Piripiri-PI Laise Do Nascimento Silva, Elane Dos Santos Silva Barroso, Geane Maria Oliveira Lima, Jane Gabriela Soares de Lemos, Edvaldo Pio da Silva

$\mathrm{Na}$ figura 02, observa-se a apresentação acerca dos canais de comunicação e as estratégias consideradas como vantagens competitivas para as empresas pesquisadas.

\begin{tabular}{|c|c|c|c|c|c|}
\hline Canal da CIM mais rentável & $\%$ & $\begin{array}{c}\text { Controle sobre o no } \\
\text { de clientes }\end{array}$ & $\%$ & $\begin{array}{c}\text { Satisfação dos } \\
\text { Clientes }\end{array}$ & $\%$ \\
\hline Merchandising & $20 \%$ & Possui & $80 \%$ & Satisfeitos & $80 \%$ \\
\hline Marketing Pessoal & $20 \%$ & Não Possui & $20 \%$ & Insatisfeitos & $20 \%$ \\
\hline Marketing Direto & $20 \%$ & --------- & --- & ---------- & --- \\
\hline Promoção e Eventos & $20 \%$ & ---------- & --- & ---------- & --- \\
\hline Propaganda e Publicidade & $20 \%$ & ---------- & --- & --------- & --- \\
\hline
\end{tabular}

Figura 2. Canais da CIM

Fonte: Dados da Pesquisa (2020).

Quanto ao canal de comunicação de maior retorno financeiro, totalizando $20 \%$ das clínicas consideraram Merchandising, Marketing Pessoal, Marketing Direto, Promoção e Eventos, Propaganda e Publicidade. Sobre essa perspectiva, Seta et al. (2016) declaram que comunicar-se diretamente com o cliente-alvo sem intermediários, por meio de e-mails, telefonemas, correios podem trazer resultados mais satisfatórios para a empresa. Tais canais contribuem para atingir a população em massa, ou seja, mostrar a imagem da empresa no mercado a fim de se alcançar determinado posicionamento.

As clínicas de fisioterapia e estética participantes do estudo pontuaram que atuam no mercado entre 5 a 10 anos e que para se posicionarem nesse ramo competitivo precisam elaborar métodos para fidelizar seus clientes-alvo e conseguir mais clientes potenciais.

De acordo com a pesquisa quanto às estratégias de vantagem competitiva $60 \%$ disseram que para se destacar no mercado buscam conquistar mais clientes por meio de redução no preço dos serviços, visto que essa maneira é percebida como a mais eficaz no mercado local onde se concentram. Além disso, optam por manter a fidelidade 
Gestão de Custos com Comunicação Integrada de Marketing nas Clínicas de Fisioterapia e Estética no Município de Piripiri-PI Laise Do Nascimento Silva, Elane Dos Santos Silva Barroso, Geane Maria Oliveira Lima, Jane Gabriela Soares de Lemos, Edvaldo Pio da Silva

dos clientes oferecendo promoções de eventos, como palestras, reuniões, coquetéis, experiências acrescidas de serviços oferecidos por profissionais de qualidade.

Na visão de Queiroz et al. (2018), a CIM deve ser a mais adequada possível no âmbito das organizações sendo meio estratégico atuante na função comunicação, cujo objetivo principal consiste em alavancar a marca dos produtos e serviços ofertados de forma a construir uma identidade positiva no mercado.

Com relação ao controle sobre o crescimento no número de clientes e os benefícios ofertados, $80 \%$ dos gestores disseram que possuem esse controle e afirmaram que os clientes-alvo estão cientes dos benefícios que recebem, divergindo dos $20 \%$ que afirmaram não realizar ou saber a satisfação dos clientes diante dos serviços ofertados. Inteirar-se sobre os anseios dos clientes e buscar controle da aquisição desses pelas empresas caracteriza-se de suma importância para que esta tenha um melhor direcionamento sobre as atividades e resultados.

Assim, destaca-se como é importante o conhecimento dos clientes participantes de qualquer organização, já que ao consumir produtos ou serviços de uma empresa específica, denota-se certo nível de confiança e credibilidade na reputação que a empresa possui. Por outro lado, contatar o cliente e receber feedback sobre sua satisfação, bem como estar ciente dos benefícios ofertados contribui para detectar quais melhorias podem estar sendo realizadas a fim de qualificar os serviços prestados (Garcia \& Mainardes, 2016).

As ferramentas da CIM propiciam o atendimento às necessidades de aquisição de bens e serviços que na maioria dos casos estão apoiados a fatores demográficos, psicográficos participantes da determinação de compra dos consumidores. Daí, a compreensão da potencialização encontrada nos canais da comunicação integrada quando aplicados eficazmente, pois pode favorecer vantagens competitivas. Logo, o valor do cliente tem se tornado uma temática de cunho enriquecedor, haja vista que a sua avaliação se constitui de fundamental relevância para a manutenção da empresa sob a ótica de sua lucratividade. Tanto a criação do valor para o cliente como o 
Gestão de Custos com Comunicação Integrada de Marketing nas Clínicas de Fisioterapia e Estética no Município de Piripiri-PI Laise Do Nascimento Silva, Elane Dos Santos Silva Barroso, Geane Maria Oliveira Lima, Jane Gabriela Soares de Lemos, Edvaldo Pio da Silva

crescimento da organização devem estar incorporados no planejamento estratégico e em todo processo de venda (Queiroz et al., 2018).

As organizações vivem em um contexto de agressividade no mercado e a todo instante novas ideias surgem, empresas se instalam apresentando novidades que comprometem as demais empresas já existentes. Para se manter em um meio marcado por diversas transformações é necessário que as organizações olhem com mais veemência para os seus clientes, sempre buscando apresentar a qualidade, confiança e valor.

A seção seguinte apresenta informações sobre a análise de dados do uso da contabilidade de custos pelas clínicas de fisioterapia e estética do lócus pesquisado.

\subsection{Aplicabilidade da Contabilidade de Custos}

A contabilidade de custo corresponde a uma área do conhecimento que contempla a produção de dados úteis para a formação de informações acerca dos custos despendidos para auxílio na tomada de decisão.

Dessa forma, a figura 03 apresenta dados da contabilidade de custos e a utilização de sistema para registro de informações:

\begin{tabular}{|c|c|c|c|}
\hline Conhecimento sobre Contabilidade de Custos & $\%$ & Uso de Sistemas & $\%$ \\
\hline Pouco & $0 \%$ & Sim & $0 \%$ \\
\hline Razoável & $80 \%$ & Não & $100 \%$ \\
\hline Nenhum & $20 \%$ & ------- & \\
\hline
\end{tabular}

Figura 3. Conhecimento sobre Contabilidade de Custos e Uso de Sistemas

Fonte: Dados da Pesquisa (2020).

A respeito do conhecimento sobre aspectos conceituais da contabilidade e uso de sistema para o gerenciamento de custos, $80 \%$ informou ter um conhecimento razoável e $100 \%$ dos gestores declararam não ter um sistema que os auxiliam na realização desse controle. 
Gestão de Custos com Comunicação Integrada de Marketing nas Clínicas de Fisioterapia e Estética no Município de Piripiri-PI Laise Do Nascimento Silva, Elane Dos Santos Silva Barroso, Geane Maria Oliveira Lima, Jane Gabriela Soares de Lemos, Edvaldo Pio da Silva

De acordo com Bergmann et al. (2019), a missão do controle consiste em fornecer dados úteis no controle orçamentário, a fim de possibilitar para a organização uma previsão de suas operações e registrar as ocorrências diárias para avaliação e comparação com períodos definidos. Sendo fundamental não somente para maximizar os lucros, aumentar a produtividade e reduzir custos, mas para o acompanhamento de informações. A figura 04 notifica dados em relação ao processo de realização do controle de custos:

\begin{tabular}{|c|c|c|c|}
\hline Responsável pelo Controle de Custos & $\%$ & $\begin{array}{c}\text { Ferramenta utilizada no Controle de } \\
\text { Custos }\end{array}$ & $\%$ \\
\hline Contador & $20 \%$ & Planilhas no Excel & $40 \%$ \\
\hline Gestor & $20 \%$ & Caderno de Anotação & $60 \%$ \\
\hline Proprietário & $60 \%$ & Sistemas & $0 \%$ \\
\hline
\end{tabular}

Figura 4. Gerenciamento dos Custos

Fonte: Dados da Pesquisa (2020).

Os resultados apontaram que $60 \%$ dos gestores fazem o próprio controle de custo e apenas $20 \%$ deles têm o auxílio de um contador. Nota-se que essas clínicas controlam os custos de modo rústico, o que pode vir a ser um grande problema futuro para a organização. Isso porque, como mencionado, a maioria dos gestores não contam com profissionais aptos para a função contábil e nem possuem sistemas que Ihes auxiliem.

No tocante à necessidade de alteração metodológica no controle dos custos, cerca de $80 \%$ afirmou que há uma necessidade de mudança na metodologia desse controle em seu empreendimento e que realizam esse controle em periodicidade mensal.

Salienta-se que toda empresa precisa acompanhar suas atividades de custeio, tendo assim controle sobre os dispêndios dos seus produtos e serviços, independentemente do porte estrutural ou financeiro. Para tanto, torna-se 
Gestão de Custos com Comunicação Integrada de Marketing nas Clínicas de Fisioterapia e Estética no Município de Piripiri-PI Laise Do Nascimento Silva, Elane Dos Santos Silva Barroso, Geane Maria Oliveira Lima, Jane Gabriela Soares de Lemos, Edvaldo Pio da Silva

imprescindível entender a correta definição de que o custo se refere ao conjunto de gastos com materiais participantes das atividades principais de uma organização para a produção de bens e serviços e que as despesas são gastos característicos de atividades administrativas (Lopes \& Beuren, 2017).

O sucesso organizacional depende da forma como as empresas gerenciam seus custos, controlam as atividades e mantêm-se atualizadas por meio do registro de dados das ocorrências diárias. Assim, para que se tenha sustentação essas devem ter consigo o maior número de dados reais tanto para obtenção de informações precisas como para melhor clareza da situação e evolução da empresa.

$\mathrm{Na}$ figura 05 , visualiza-se a situação da separação entre custos e despesas de acordo com as empresas pesquisadas e a aplicação de gastos com a Comunicação Integrada de Marketing:

\begin{tabular}{|c|c|c|c|c|c|}
\hline $\begin{array}{c}\text { Separação Entre Custos } \\
\text { e Despesas }\end{array}$ & $\%$ & $\begin{array}{c}\text { Classificação dos } \\
\text { Gastos com CIM }\end{array}$ & $\%$ & $\begin{array}{c}\text { Rentabilidade dos } \\
\text { gastos com CIM }\end{array}$ & $\%$ \\
\hline Realiza & $60 \%$ & Despesa & $0 \%$ & Rentáveis & $80 \%$ \\
\hline Não Realiza & $40 \%$ & Investimento & $100 \%$ & Não rentáveis & $20 \%$ \\
\hline
\end{tabular}

Figura 5. Separação e Classificação dos Custos

Fonte: Dados da Pesquisa (2020).

Quando questionados sobre a separação entre custos e despesas $60 \%$ dos gestores declaram que há essa distinção, comprovando que fazer essa diferenciação propicia um melhor controle sobre os recursos da empresa. No entanto, vale dizer que custos e despesas ainda que vistos com equivalência possuem conceitos divergentes. O primeiro está ligado a tudo aquilo que se volta para a produção de bens e serviços, como já declarado anteriormente. O segundo refere-se a gastos de natureza administrativa. Tais definições apoiam o registro contábil dos recursos úteis nos processos produtivos e gerenciais para elaboração do balanço financeiro com foco na tomada de decisão (Crepaldi \& Crepaldi, 2018). 
Gestão de Custos com Comunicação Integrada de Marketing nas Clínicas de Fisioterapia e Estética no Município de Piripiri-PI Laise Do Nascimento Silva, Elane Dos Santos Silva Barroso, Geane Maria Oliveira Lima, Jane Gabriela Soares de Lemos, Edvaldo Pio da Silva

Quanto a classificação dos gastos realizados com a Comunicação Integrada de Marketing $100 \%$ dos respondentes consideram um investimento, porém $20 \%$ dessas clínicas realçam que os gastos feitos com divulgação não são rentáveis e nem geram impactos positivos nos resultados da empresa. Nota-se uma contradição nas respostas, uma vez que os gestores percebem os gastos feitos com CIM como investimento, porém parte deles ainda não obtém um retorno considerado satisfatório. E em situações de crise econômica as clínicas afirmam não realizarem gastos em estratégias de Comunicação Integrada de Marketing.

Para Sarquis et al. (2019), os recursos aplicados em marketing são investimento e não despesas. Esses investimentos não se referem apenas à propaganda e publicidade de seus produtos ou serviços, mas, também a toda e qualquer ação que o gestor realize para atrair mais clientes. Verifica-se que parte dos respondentes reconhecem a Comunicação Integrada de Marketing e a contabilidade de custos como algo importante na organização. Porém, a CIM ainda é vista como atividade secundária sendo apenas um acréscimo ao negócio e não parte dele.

\section{CONSIDERAÇÕES FINAIS}

Os resultados obtidos apontam que o dispêndio com as ferramentas de marketing na visão dos gestores das clínicas de fisioterapia e estética de Piripiri representa um investimento quando ocorre a saída dos recursos. Cerca de $80 \%$ declarou obter retorno sobre essa aplicação e $20 \%$ julga não obter o resultado esperado em cima do que foi gasto, deixando de ser um investimento atrativo diante de sua baixa performance.

Observa-se que a Comunicação Integrada de Marketing na visão dos gestores é um instrumento importante e que a falta desses elementos implica na redução das vendas, perda de clientes e mercado, sendo a propaganda o item mais utilizado. Quanto às estratégias utilizadas para se destacar no mercado, os dados apontam que 
Gestão de Custos com Comunicação Integrada de Marketing nas Clínicas de Fisioterapia e Estética no Município de Piripiri-PI Laise Do Nascimento Silva, Elane Dos Santos Silva Barroso, Geane Maria Oliveira Lima, Jane Gabriela Soares de Lemos, Edvaldo Pio da Silva

as clínicas buscam fidelizar e conquistar mais clientes a partir do momento que oferecem promoções no valor dos serviços.

Os gestores compreendem que a execução das ações de marketing aumenta os custos. Na verdade, se olhar para a realidade que vivenciam no mercado atual uma Comunicação Integrada de Marketing estratégica e bem executada pode alavancar a empresa, melhorar seu posicionamento, atrair e fidelizar clientes e trazer grande retorno financeiro, visto que a Comunicação Integrada de Marketing torna-se um investimento quando aplicada eficazmente pela empresa, auxiliando-a na elaboração de estratégias e tomada de decisões assertivas.

Constatou-se a necessidade de um controle de custos mais eficaz com um maior auxílio da contabilidade já que esse controle ainda é realizado de uma maneira arbitrária, pouco munido de informações contábeis, sendo feito pelos próprios gestores.

Dessa forma, considera-se que este estudo traz contribuições para os discentes e docentes do curso de administração, pois apresenta uma aplicação prática das ferramentas de marketing e contabilidade de custos nas clínicas locais além de ser um tema pouco investigado.

Logo, endossa-se a importância da Comunicação Integrada de Marketing para as referidas empresas por possibilitar um conjunto articulado de esforços, ações, estratégias e produtos de comunicação, planejados e desenvolvidos para agregar valor à sua marca ou de consolidar a sua imagem. Enquanto limitações tem-se a investigação em apenas um ramo de atuação ao tempo que oportuniza pesquisas futuras para expansão do estudo em outros setores e a adoção de outros métodos de pesquisa.

\section{REFERÊNCIAS}

Antônio, R. M., Rathke, A. A. T., Moraes, B. C., \& Ambrozini, M. A. (2018). O efeito do volume de negócios nas opções de recomendação de ações dos analistas. Brazilian Review Of Finance, 15 (2), 287-311. Doi: 
Gestão de Custos com Comunicação Integrada de Marketing nas Clínicas de Fisioterapia e Estética no Município de Piripiri-PI Laise Do Nascimento Silva, Elane Dos Santos Silva Barroso, Geane Maria Oliveira Lima, Jane Gabriela Soares de Lemos, Edvaldo Pio da Silva

http://dx.doi.org/10.12660/rbfin.v15n2.2017.67390.

Breda, F., Cruz, C. M. L., Hermes, L. C. R., \& Medeiros, J. F. (2020). Plano de Desenvolvimento Institucional em Instituições de Ensino Superior Brasileiras e a Gestão da Comunicação Integrada de Marketing no Ambiente On-Line. Desenvolvimento em Questão, 18(51), 331-354.

Barboza, S., \& Silva, W. (2019). Contribuições do Marketing Social na Formação do Administrador. Administração: Ensino e Pesquisa, 20(2), 286-318.

Bergmann, V. A., Gresele, W. D., \& Walter, S. A. (2019). Análise de custos de uma empresa de doces artesanais de Marechal Cândido Rondon, Paraná. Revista de Gestão, Finanças e Contabilidade, 8(2), 155-173.

Crepaldi, S. A., \& Crepaldi, G. S. Contabilidade de Custos. 06 ed. São Paulo: Atlas, 2018.

Diniz, F., \& Suarez, M. C. (2018). Cultural meanings and consumers' discourses about their brand abandonment. Brazilian Administration Review, 15(1), e170061. https://doi.org/10.1590/1807-7692bar2018170061.

Exame. Mercado de estética cresce junto com as mulheres. 2017. Disponível em: https://exame.abril.com.br/negocios/dino/mercado-de-estetica-cresce-junto-comas-mulheres-shtml/. Acesso em: 20/07/2020.

Figueiredo, A. M., \& Souza, S. R. G. (2011). Como elaborar projetos, monografias, dissertações e teses: da redação científica a apresentação do texto final. $4^{\circ} \mathrm{ed}$. Rio de Janeiro: lumen juris.

Garcia, M. M., \& Mainardes, E. W. (2016). O papel da Comunicação Integrada de Marketing na decisão de compra de um imóvel. Caderno Profissional de Marketing-UNIMEP, 4(2), 1-17.

Gil, A. C. (2017) Como Elaborar Projetos de Pesquisa. (6a ed.). São Paulo, Atlas.

Kotler, P., \& Keller, K. L. (2010). Administração de marketing. 12. ed. São Paulo: Pearson Prentice Hall - capa vermelha.

Lopes, I. F., \& Beuren, I. M. (2017). Comportamento dos custos e sua relação com medidas de eficiência operacional em companhias aéreas. Revista Base (Administração e Contabilidade) da UNISINOS, 14(1), 30-46. 
Gestão de Custos com Comunicação Integrada de Marketing nas Clínicas de Fisioterapia e Estética no Município de Piripiri-PI Laise Do Nascimento Silva, Elane Dos Santos Silva Barroso, Geane Maria Oliveira Lima, Jane Gabriela Soares de Lemos, Edvaldo Pio da Silva

Marcolin, C., \& de Deus, G. (2018). Mapeamento de Processos e Contabilidade de Custos: Estudo em uma Indústria Frigorífica. ConTexto- Revista do Programa de Pós-Graduação em Controladoria e Contabilidade da UFRGS, 18(39).

Merabet, D. O. B., Silva, R. O., Gonçalves, C. P., \& Barros, D. F. (2020). Quão plural é o ensino de Marketing? Um estudo exploratório com professores dos cursos de graduação em Administração. Administração: Ensino e Pesquisa, 21(2), 1-26.

Pereira, E. S. (2015). Eventos estratégicos no composto da Comunicação Integrada: Marketing e relações públicas no fortalecimento de marcas. Caderno Profissional de Marketing-UNIMEP, 3(2), 48-58.

Petrin, R., Cardozo, E. A. A., \& Christino, J. M. M. (2019). Fatores que impactam a intenção de compra online. Caderno Profissional de Marketing-UNIMEP, 7(2), 101114.

Queiroz, R. S. B., Queiroz, M. J., Yanaze, M. H., \& Mazieri, M. R. (2018). Modelo conceitual para avaliação da comunicação de marketing em franquias de bens de consumo. Revista Brasileira de Marketing, 17(2), 264-277.

Sarquis, A. B., Hoeckesfeldet, L., Cittadin, J., \& Lenzi, F. C. (2019). Práticas Contemporâneas de marketing na perspectiva relacional em empresas de serviços de contabilidade. Revista Contabilidade, Gestão e Governança, 22(1), 59-84.

Sebrae. Serviço Brasileiro de Apoio às Micro e Pequenas Empresas. MEl do mercado de beleza cresce $567 \%$ em 5 anos, 2016. Disponível em: http://agenciasebrae.com.br/sites/asn/uf/NA/mei-do-mercado-de-beleza-cresce567-em-5-anos,ebb54341dedbc410VgnVCM2000003c74010aRCRD. Acesso em: $01 / 10 / 2020$.

Seta, R. C., Mainardes, E. W., \& Silva, E. R. T. (2016). Ações de Marketing de Relacionamento Utilizadas pelas Agências de Publicidade e Propaganda. Revista Brasileira de Marketing, v. 15, n. 2, p. 281-296.

Silva, G. E., Pitombo, T. D. T., \& Zaccaria, R. B. (2017). CIM em instituições de ensino e a evolução do composto de marketing. Caderno Profissional de MarketingUNIMEP, 5(1), 15-30.

Silva, M. J. B., Barbosa, M. L. A., Costa, M. F., \& Gomes, J. P. (2020). Entre posse e acesso: compreendendo a extensão do self no consumo colaborativo. Organizações \& Sociedade, 27(93). 
Gestão de Custos com Comunicação Integrada de Marketing nas Clínicas de Fisioterapia e Estética no Município de Piripiri-PI

Laise Do Nascimento Silva, Elane Dos Santos Silva Barroso, Geane Maria Oliveira Lima, Jane Gabriela

Soares de Lemos, Edvaldo Pio da Silva

Data de Submissão: 05/10/2020

Data de Aceite: 15/12/2020 